\title{
S-Sm (Sulfur-Samarium)
}

\author{
H. Okamoto
}

The Sm-S phase diagram in [Massalski2] was based on [Moffatt] with a minor modification for phase rule consistency.

The S-richest intermediate phase shown in this phase diagram was $\mathrm{SmS}_{2}$. Its crystal structure could not be well defined.

[2006Vas] discovered that there are three phases $\mathrm{Sm}_{7} \mathrm{~S}_{13}$, $\mathrm{Sm}_{9} \mathrm{~S}_{17}$ and $\mathrm{Sm}_{10} \mathrm{~S}_{19}$ instead of $\mathrm{SmS}_{2}$. The [Massalski2] phase diagram was modified in Fig. 1 to reflect this discovery.
Table 1 shows $\mathrm{Sm}-\mathrm{S}$ crystal structure data.

\section{Reference}

2006Vas: I.G. Vasil'eva, Phase Equilibrium and $\mathrm{p}_{\mathrm{s}}-\mathrm{T}-\mathrm{x}$ Diagrams of the $\mathrm{LnS}_{3}-\mathrm{LnS}_{2}(\mathrm{Ln}=\mathrm{La}, \mathrm{Pr}, \mathrm{Nd}, \mathrm{Sm}-\mathrm{Er})$ Systems, Zh. Fiz. Khim., 2006, 80(11), p 2068-2073, in Russian; TR: Russ. J. Phys. Chem., 2006, 80(11), p 1842-1847

Table 1 Sm-S crystal structure data

\begin{tabular}{|c|c|c|c|c|c|}
\hline Phase & Composition, at. $\% \mathrm{~S}$ & Pearson symbol & Space group & Strukturbericht designation & Prototype \\
\hline$(\gamma \mathrm{Sm})$ & 0 & $c 12$ & $\operatorname{Im} \overline{3} m$ & $A 2$ & W \\
\hline$(\beta \mathrm{Sm})$ & 0 & $h P 2$ & $\mathrm{P6}_{3} / m m c$ & $A 3$ & $\mathrm{Mg}$ \\
\hline$(\alpha \mathrm{Sm})$ & 0 & $h R 3$ & $R \overline{3} m$ & $\ldots$ & $\alpha \mathrm{Sm}$ \\
\hline $\mathrm{SmS}$ & 50 & $c F 8$ & $F m \overline{3} m$ & $B 1$ & $\mathrm{NaCl}$ \\
\hline $\mathrm{Sm}_{3} \mathrm{~S}_{4}$ & 57.1 & $c I 28$ & $I \overline{4} 3 d$ & $D 7_{3}$ & $\mathrm{Th}_{3} \mathrm{P}_{4}$ \\
\hline $\mathrm{Sm}_{5} \mathrm{~S}_{7}$ & 58.3 & $t I 192$ & $I 4_{1} /$ acd & $\ldots$ & $\ldots$ \\
\hline $\mathrm{Sm}_{2} \mathrm{~S}_{3}$ & 60 & $o P 20$ & Pnma & $\ldots$ & $\ldots$ \\
\hline $\mathrm{Sm}_{7} \mathrm{~S}_{13}$ & 65 & $\ldots$ & $\cdots$ & $\cdots$ & $\ldots$ \\
\hline $\mathrm{Sm}_{9} \mathrm{~S}_{17}$ & 65.4 & $\ldots$ & $\ldots$ & $\ldots$ & $\ldots$ \\
\hline $\mathrm{Sm}_{10} \mathrm{~S}_{19}$ & 65.5 & $\ldots$ & $\ldots$ & $\ldots$ & $\ldots$ \\
\hline$(\beta S)$ & 100 & $m P 48$ & $P 2_{1} / \mathrm{a}$ & $\ldots$ & $\ldots$ \\
\hline$(\alpha S)$ & 100 & $o F 128$ & Fddd & $A 16$ & $\alpha \mathrm{S}$ \\
\hline
\end{tabular}

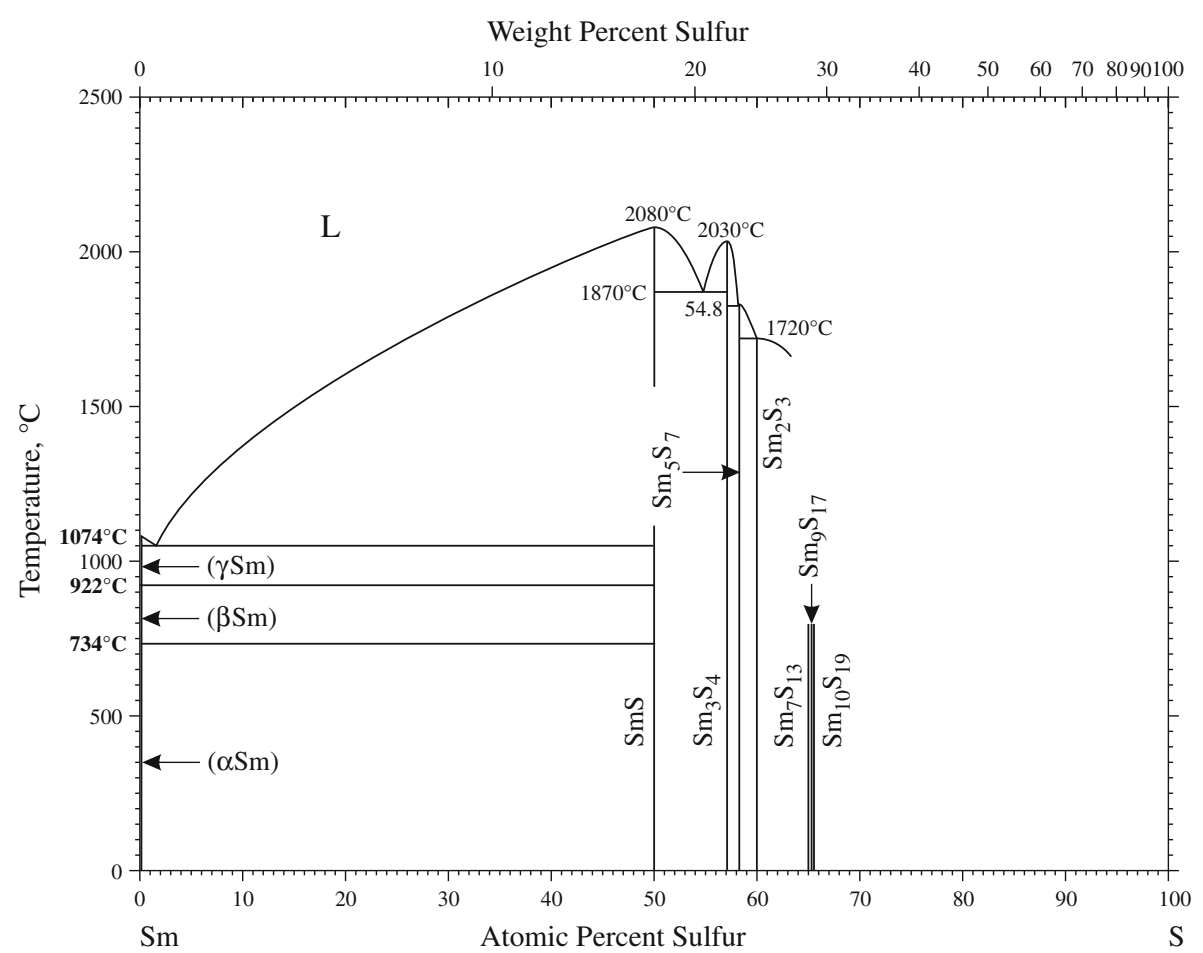

Fig. 1 Sm-S phase diagram 